\title{
Annular gap model for multi-wavelength pulsed emission from young and millisecond pulsars
}

\author{
Yuan Jie $\mathrm{Du}^{1}$ and Guo Jun Qiao ${ }^{2}$ \\ ${ }^{1}$ National Space Science Center, Chinese Academy of Sciences, \\ NO.1 Nanertiao, Zhongguancun, Haidian district, Beijing 100190, China \\ email: duyj@nssc.ac.cn \\ ${ }^{2}$ School of Physics, Peking University, Beijing 100871, China \\ email: gjn@pku.edu.cn
}

\begin{abstract}
The multi-wavelength pulsed emission from young pulsars and millisecond pulsars can be well modeled with the single-pole 3-dimension annular gap and core gap model. To distinguish our single magnetic pole model from two-pole models (e.g. outer gap model and two-pole caustic model), the convincing values of the magnetic inclination angle and the viewing angle will play a key role.
\end{abstract}

Keywords. gamma rays: stars, pulsars: general, radiation mechanisms: non-thermal

\section{Introduction}

Pulsars are fascinating astronomical objects in the universe. Many pulsars, including young normal pulsars and millisecond pulsars (MSPs), radiate multi-wavelength pulsed emission, which have not been completely understood.

High energy emission (e.g. $\gamma$-ray emission) from pulsars takes away a significant fraction of the rotational energy. Thanks to the launching of Astro-rivelatore Gamma a Immagini LEggero (AGILE) and the Fermi Gamma-ray Space Telescope (FGST), more than one hundred new $\gamma$-ray pulsars have been discovered in the last year, including gamma-ray only pulsars and a new population of millisecond pulsars (Abdo et al. 2009, Abdo et al. 2010a, Pellizzoni et al. 2009). From observations, MSPs are analogous to young pulsars, which have multi-wavelength pulsed emission from radio $\left(10^{-6} \mathrm{eV}\right)$ to $\gamma$-ray band. Do MSPs and young pulsars share a simple model that contains simlar emission region and acceleration mechanism to self-consistently explain their multi-wavelength emission?

Some models have been proposed to explain pulsar's multi-wavelength emission, as we will summarize below, differing on the acceleration region of the primary particles and the mechanism for the production of the high energy photons. Initially aiming to explain the high-energy pulsed emission from young pulsars, four traditional physical or geometrical magnetospheric models which have been proposed to explain pulsed $\gamma$ ray emission of pulsars: the polar cap model (Daugherty \& Harding 1994), the outer gap model (Cheng, Ho \& Ruderman 1986), TPC/slot gap model (Dyks \& Rudak 2003). The distinguishing features of these pulsar models are different acceleration electric field regions for primary particles and relevant emission mechanisms to radiate high energy photons (Du et al. 2011, 2012). One of the key discrepancies of these emission models is the one-pole or two-pole emission pattern with two important geometry parameters: the magnetic inclination angle $\alpha$ and the view angle $\zeta$. 

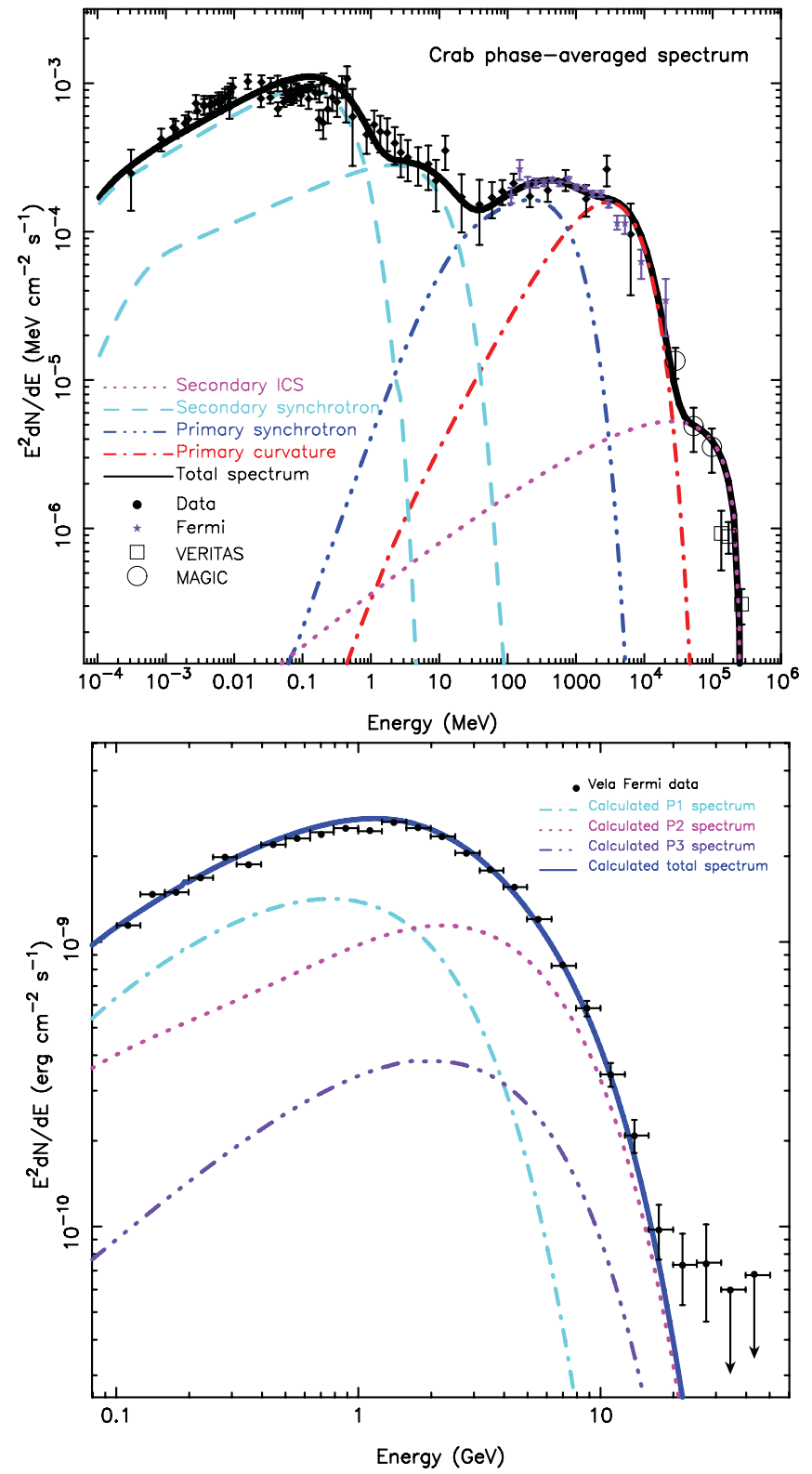

Figure 1. Upper panel: the modeled radio-to-TeV phase-averaged spectrum for the Crab pulsar (adopted from Du et al. 2012). Bottom panel: the modeled $\gamma$-ray phase-averaged spectrum for the Vela pulsar (adopted from Du et al. 2011).

The annular gap model was originally proposed by Qiao et al. (2004, 2007). The critical field lines divide the polar-cap region of a pulsar magnetosphere into two distinct parts: the core gap region and the annular gap region (Du et al. 2011, 2012). The former gap is located around the magnetic axis and within the critical field lines; the latter is located between the critical field lines and the last open field lines. The width of the annular gap region is anti-correlated with the pulsar period, it is therefore larger for pulsars with smaller spin periods (Du et al. 2010). The region for high energy emission in the annular gap model is concentrated in the vicinity of the null charge surface, i.e., an 
intermediate emission height, different from the outer gap model. The annular gap has a sufficient thickness of trans-field lines and a wide altitude range for particle acceleration. This model combines the advantages of the polar gap, the slot gap and the outer gap models, and works well for pulsars with short spin periods. It is a promising model to explain high energy emission from young and millisecond pulsars.

\section{Modeled Results of Pulse Profiles and Spectra}

A convincing model should have a simple clear emission geometric picture with reasonable input parameters, which can not only reproduce multi-wavelength light curves for young pulsars but also for MSPs. Here we will briefly introduce our modeled results of pulse profiles and phase-averaged spectrum for the Vela pulsar, Crab pulsar and some millisecond pulsars. The detailed calculations in the annular gap model can be found in Du et al. $(2010,2011,2012)$.

We can solve the problems of the third peak (P3) in the $\gamma$-ray pulse profiles and the emission mechanism of $\mathrm{GeV}$ band for the Vela pulsar (Du et al. 2011; as shown in bottom panel of Figure 1). The GeV band emission from the Vela pulsar is originated mainly from Synchro-curvature radiation (Zhang \& Cheng 1995) from primary particles, while synchrotron radiation from secondary particles have some contributions to the low-energy $\gamma$-ray band (e.g., $0.1-0.3 \mathrm{GeV}$ ). Meanwhile, the total spectrum (thick black solid line in top panel of Figure 1) is calculated in the annular gap model. It is found that the curvature radiation and synchrotron radiation from primary particles is mainly contributed to $\gamma$-ray band $(20 \mathrm{MeV}$ to $20 \mathrm{GeV})$; synchrotron radiation from CR-induced pairs and ICS-induced pairs dominates the X-ray band and soft $\gamma$-ray band $(100 \mathrm{eV}$ to $10 \mathrm{MeV})$. ICS from the pairs contributes to hard TeV $\gamma$-ray band $(\sim 20 \mathrm{GeV}$ to $400 \mathrm{GeV})$.

\section{Conclusion}

The annular gap model is a self-consistent single-pole model not only for young pulsars (Du et al. 2011, 2012), but also for MSPs (Du et al. submitted). Multi-wavelength pulsed emission can be well explained by this model.

\section{References}

Abdo, A. A., Ackermann, M., Ajello, M., et al. 2009, Science, 325, 848

Abdo, A. A., et al. 2010a, ApJs, 187, 460

Cheng, K. S., Ho, C., \& Ruderman, M. 1986, ApJ, 300, 500

Daugherty, J. K. \& Harding, A. K. 1994, ApJ, 429, 325

Du, Y. J., Qiao, G. J., Han, J. L., Lee, K. J., \& Xu, R. X. 2010, MNRAS, 406, 2671

Du, Y. J., Han, J. L., Qiao, G. J., \& Chou, C. K. 2011, ApJ, 731, 2

Du, Y. J., Qiao, G. J., \& Wang, W. 2011, ApJ, 748, 84

Dyks, J. \& Rudak, B. 2003, ApJ, 598, 1201

Zhang, J. L. \& Cheng, K. S. 1995, Physics Letters A, 208, 47

Pellizzoni, A., et al. 2009, ApJ, 695, L115

Qiao, G. J., Lee, K. J., Wang, H. G., Xu, R. X., \& Han, J. L. 2004, ApJ, 606, L49

Qiao, G. J., Lee, K. J., Zhang, B., Wang, H. G., \& Xu, R. X. 2007, Chin. J. Astron. Astrophys., 7,496 